Chirurg 2013 $\cdot 84: 234$

DOI 10.1007/s00104-013-2465-4

Online publiziert: 13. Februar 2013

๑) Springer-Verlag Berlin Heidelberg 2013

J. Reibetanz · C.-T. Germer

Klinik für Allgemein-, Viszeral-, Gefäß- und Kinderchirurgie, Universitätsklinikum Würzburg

\title{
Laparoskopische vs. offene Chirurgie beim kolorektalen Karzinom
}

\section{0-Jahres-Ergebnisse des CLASICC-Trial}

\section{Originalpublikation}

Green BL, Marshall HC, Collinson F et al (2013) Long-term follow-up of the Medical Research Council CLASICC trial of conventional versus laparoscopically assisted resection in colorectal cancer. Br J Surg 100:75-82

\section{Hintergrund}

Die Laparoskopie findet zunehmend auch in der onkologischen Kolon- und Rektumchirurgie Anwendung. Ziel des CLASICC-Trial (Conventional versus Laparoscopic-Assisted Surgery in Colorectal Cancer) war die Evaluierung der Sicherheit und onkologischen Gleichwertigkeit des laparoskopischen Zugangs beim kolorektalen Karzinom (CRC) verglichen mit der konventionellen Chirurgie.

\section{Methode}

Der CLASICC-Trial ist eine multizentrische, randomisiert-kontrollierte Studie, in die zwischen 1996 und 2002 insgesamt 794 Patienten mit Kolonkarzinom $(\mathrm{n}=413)$ oder Rektumkarzinom $(\mathrm{n}=381)$ eingeschlossen wurden. In einer 2:1-Randomisierung erfolgte die Resektion entweder mittels laparoskopisch-assistiertem $(n=526)$ oder offenem ( $n=268)$ Zugang. Die Vergleichbarkeit beider Zugangswege hinsichtlich des perioperativen Outcomes und der mittel- und langfristigen onkologischen Ergebnisse wurden bereits in früheren Publikationen bestätigt $[1,2]$. Aktuell werden die 10-Jahres-Ergebnisse des CLASICC-Trial berichtet.

\section{Ergebnisse}

Das mediane Follow-up betrug für alle noch lebenden Patienten 91,8 Monate (74-112), 428 Patienten waren innerhalb des Beobachtungszeitraums verstorben $(\mathrm{n}=144$ in der offenen und $\mathrm{n}=284$ in der laparoskopischen Gruppe). Hinsichtlich des Gesamtüberlebens (78,3 [95\%-Konfidenzintervall $65,8-106,6]$ vs. 82,7 [69,194,8] Monate) und des krankheitsfreien Überlebens (89,5 [67,1-121,7] vs. 77,0 [63,3-94,0] Monate) zeigten sich keine signifikanten Unterschiede zwischen laparoskopischer und offener Chirurgie. Das Auftreten einer metachronen Metastasierung war ebenfalls unabhängig vom Zugang (19,8\% in der offenen und 22,7\% in der laparoskopischen Gruppe). Gleiches galt auch für das Risiko eines Lokalrezidivs, wobei insgesamt rechtsseitige Kolonkarzinome eher zu Lokalrezidiven neigten als linksseitige (14,7\% vs. $5,2 \%$, $\mathrm{p}=0,019$ ).

\section{Diskussion und Fazit}

Das 10-Jahres-Ergebnis des CLASICC-Trial ist beeindruckend eindeutig: Die onkologischen Resultate nach offener oder laparoskopischer Chirurgie des CRC sind langfristig grundsätzlich vergleichbar, dies bestätigt die bereits publizierten onkologischen 3- und 5-Jahres-Resultate von CLASICC [1, 2]. Zusammen mit der Überlegenheit der Laparoskopie hinsichtlich der Kurzzeitergebnisse wird diese daher als „Therapie der Wahl“ beim CRC propagiert! Subgruppenspezifisch und stadienabhängig kann das Outcome von laparoskopisch und offen operier- ten Patienten jedoch divergieren: So zeigt die detaillierte Analyse eine Verlängerung des Gesamtüberlebens für offen operierte Patienten mit lokal fortgeschrittenen Kolonkarzinomen im Stadium III gegenüber der Laparoskopie (79 vs. 35 Monate, $p=0,031$ ). Für die Subgruppe der Kolonkarzinome - nicht jedoch für das Rektumkarzinom - konnte erneut der negative prognostische Einfluss der intraoperativen Konversion (laparoskopisch $\rightarrow$ offen) aufgezeigt werden, jeweils mit einer Verdopplung des Risikos für ein Tumorrezidiv bzw. tumorbedingtes Versterben. Auch im Licht einer zunehmend erdrückenden Evidenz für die Laparoskopie beim CRC scheint immer noch eine individuelle Indikationsstellung der angemessenste „, $\mathbf{Z u -}$ gangsweg".

\section{Korrespondenzadresse}

Prof. Dr. C.-T. Germer

Klinik für Allgemein-, Viszeral-, Gefäß- und Kinderchirurgie, Universitätsklinikum Würzburg, Oberdürrbacher Str. 6, 97080 Würzburg germer_c@klinik.uni-wuerzburg.de

Interessenkonflikt. Der korrespondierende Autor gibt für sich und seinen Koautor an, dass kein Interessenkonflikt besteht.

\section{Literatur}

1. Jayne DG, Guillou PJ, Thorpe H et al (2007) UK MRC CLASICC Trial Group. Randomized trial of laparoscopic-assisted resection of colorectal carcinoma: 3-year results of the UK MRC CLASICC Trial Group. J Clin Oncol 25:3061-3068

2. Jayne DG, Thorpe HC, Copeland J et al (2010) Fiveyear follow-up of the Medical Research Council CLASICC trial of laparoscopically assisted versus open surgery for colorectal cancer. Br J Surg 97:1638-1645 